Relations industrielles

Industrial Relations

\title{
La violation de la convention collective peut-elle donner ouverture à des dommages-intérêts?
}

\section{Pierre Verge}

Volume 26, numéro 1, 1971

URI : https://id.erudit.org/iderudit/028196ar

DOI : https://doi.org/10.7202/028196ar

Aller au sommaire du numéro

Éditeur(s)

Département des relations industrielles de l'Université Laval

ISSN

0034-379X (imprimé)

1703-8138 (numérique)

Découvrir la revue

Citer cet article

Verge, P. (1971). La violation de la convention collective peut-elle donner ouverture à des dommages-intérêts? Relations industrielles / Industrial Relations, 26(1), 234-240. https://doi.org/10.7202/028196ar

\section{Résumé de l'article}

L'exécution en nature de la convention collective n'étant pas toujours praticable, l'octroi d'une compensation pécuniaire peut être la seule façon de faire justice. Cette réponse affirmative pose le problème de la détermination de l'instance habilitée à ordonner le paiement de dommages-intérêts.
Tous droits réservés (C) Département des relations industrielles de l'Université Laval, 1971
Ce document est protégé par la loi sur le droit d'auteur. L’utilisation des services d'Érudit (y compris la reproduction) est assujettie à sa politique d'utilisation que vous pouvez consulter en ligne.

https://apropos.erudit.org/fr/usagers/politique-dutilisation/ 


\title{
La violation de la convention collective peut-elle donner ouverture à des dommages-intérêts ?
}

\author{
Pierre Verge
}

\begin{abstract}
L'exécution en nature de la convention collective n'étant pas toujours praticable, l'octroi d'une compensation pécuniaire peut être la seule façon de faire justice. Cette réponse affirmative pose le problème de la détermination de l'instance habilitée à ordonner le paiement de dommagesintérêts.
\end{abstract}

Généralement, l'arbitre des griefs ordonnera à la partie en défaut de se conformer à la convention de poser l'acte qu'elle aurait initialement dû poser, de rétablir l'ordre de la convention collective qu'elle se devait d'observer. Cependant, il est d'abord des situations où cette réparation en nature n'est pas adéquate. La remise en retard d'une somme due selon la convention ne saurait donner pleinement satisfaction au créancier, à moins que l'on ne l'indemnise du retard par le paiement de l'intérêt ou dommages-intérêts moratoires ${ }^{1}$. La réparation du défaut d'accorder un travail ou une promotion selon la con'rention doit porter sur l'équivalent du salaire, ou de la majoration de ce dernier, que le salarié aurait pu gagner à compter du moment où il avait droit au travail dont il s'agit ${ }^{2}$. Parfois, même, la réparation en nature est simplement impossible. Ainsi en est-il des dommages déjà causés par le déclanchement d'une grève illégale contrairement à une clause de non-grève dans la convention ${ }^{3}$. Dans ce dernier cas, les dommages-intérêts seront compensatoires.

ADMISSIBILITÉ DES DOMMAGES-INTÉRÊTS

Que le non respect de la convention collective puisse résulter en des dommages-intérêts, on l'admet assez aisément. Ainsi, notamment, la Cour

* Pierre Verge, Faculté de Droit, Université Laval.

1 Voir au sujet des dommages-intérêts moratoires : Rousseau v. Gouvernement de la province de Québec, ministère de l'éducation, (1970) R.O.T. 524 ou (1970) S.A.G. 981 (j. H. Lande) ; affaire Syndicat des professeurs de l'État du Québec, (1968) R.D.T. 240 (j. J.L. Poliquin) ; Orphelinat de l'Immaculée Conception de Chicoutimi, D.C.D., no 828-4 (C. Bisson, pres.)

2 Compagnie Price Limitée v. Syndicat national des travailleurs de la pulpe et du papier de Kénogami Inc., (1967) R.D.T. 570 (H. Lande).

3 Fraternité internationale des ouvriers en électricité, local 568, v. BédardGirard Limitée, B.R. (M.) no 11,741, conf. un jugement du j. Mitchell C.S. (M.), en date du 27 novembre 1968, arrêt résumé à (1969) B.R. 991. Voir aussi Re Oil, Chemical and Atomic Workers and Polymer Corp. Ltd., (1968) 10 Lab. Arb. Cas. 31, B. Laskin, pres.; refus d'annuler : Re Polymer Corporation and Oil, Chemical and Atomic Workers International Union, local 16-14, 26 D.L.R. (2d) 609 (J. Mc Ruer), conf. (1961) O.R. 438 et par Imbleau v. Laskin, (1962) R.C.S. 338. 
d'Appel entérine-t-elle le juge qui avait refusé de rejeter, de façon liminaire, une action en dommages-intérêts d'un employeur contre un syndicat qui avait déclenché une grève illégale en regard de la convention ${ }^{4}$. De diverses décisions d'arbitres ou, même, de certains jugements, l'on peut dresser le bilan suivant à l'appui de l'admissibilité d'une conclusion à des dommages-intérêts.

\section{Rejet d'arguments défavorables}

- Les dommages-intérêts ne sont pas des « conditions de travail », objet unique d'une convention collective. Cet argument est fallacieux. Les dommages-intérêts n'ont pas été recherchés immédiatement par l'un des signataires de la convention. Ils ne représentent qu'une compensation pour le défaut de l'autre partie d'observer une condition de travail que stipulait la convention ${ }^{5}$.

- De la même façon faudrait-il aborder l'argument reposant sur le fait que la convention collective ne traite pas des dommages-intérêts, spécifiquement. Ceux-ci ne sont que la conséquence de l'inobservance des termes de la convention.

- Il faudrait s'en remettre aux seules sanctions pénales du Code du travail, selon lesquelles la convention a été conclue. Les dommagesintérêts n'ont rien de «primitif », faut-il ici observer, ils ne sont que compensatoires, d'une nature «civile», du moins au sens large ${ }^{6}$.

\section{Arguments favorables}

L'on rejoint ici le fondement même de la convention collective. Tantôt, on la situe dans l'arrière-plan général du régime général des obligations civiles, d'ordre contractuel(1.) ; tantôt l'on met en relief le caractère obligatoire de l'arbitrage des griefs, mode ultime et original de sanction de la convention selon toute législation du type du Code du travail (2.).

Les DOMMAges-INTÉRÊTS, RÉPARATION COURANTE DE L'INEXÉCUTION DE L'OBLIGATION CIVILE

L'article 1065 du Code civil, tout en prévoyant la réparation en nature, dans les cas qui le permettent, énonce, sans restriction, que \& toute obligation rend le débiteur passible de dommages en cas de contravention de sa part». Des arbitres se sont autorisés de ce texte pour conclure à des dommages-intérêts, cette disposition étant en quelque sorte, selon eux, sous-jacente à l'ensemble du régime des obligations civiles, y compris, de façon supplétive, à celui de la convention collective. Il s'agit, en d'autres termes, d'un principe général de droit substantif qui est appli-

4 Arrêt Bédard-Girard Limitée, ibidem.

5 Affaire Orphelinat de l'Immaculée $\lrcorner$ Conception, supra, note (1), à la page 3.

$6 \mathrm{~J}$. Mc Ruer, dans le jugement de première instance dans l'affaire Polymer, supra, note (3), à la p. 613. 
cable à cette dernière ; on l'assimile, en effet, à cet égard du moins, au contrat civil ordinaire ?

L'intérêt pour défaut de payer à l'échéance une somme d'argent, n'est qu'une application particulière de ce principe ${ }^{8}$.

LES DOMMAGES-INTÉRÊTS, MANIFESTATION DU CARACTÈRE

EXÉCUTOIRE DE LA SENTENCE DE L'ARBITRE

Plus conforme à la réalité peut-être est cette seconde façon de raisonner à l'appui de la nécessité d'une sanction efficace de la convention. Historiquement, note-t-on, le législateur, dont celui du Québec a substitué au recours à la grève l'arbitrage obligatoire des griefs. L'arbitre, plus précisément, rend une sentence finale et obligatoire, selon la loi. Or, cette décision doit être efficace; l'arbitre, compte tenu du caractère de sa sentence, se trouve investi d'un pouvoir non seulement déclaratoire, mais également de réparation. D'ailleurs, en droit, comment séparer vraiment, dans l'exercice du pouvoir juridictionnel, la phase de la constatation du manquement du stade de la réparation? Telle est la substance, notamment, de la décision des arbitres dans l'affaire Polymer ${ }^{9}$, reprise en particulier par le juge Mc Ruer, lors du contrôle de leur juridiction. Ces énoncés vaudraient, selon nous, tout aussi bien selon la loi du Québec, qu'en vertu de la loi fédérale, qui en avait été l'occasion.

Pour ce qui est de la décision des arbitres :

"It seems to this board that fundamental to any approach to the issue is some understanding of the history and purpose of resort to "final " arbitration, to use the terms which appear respectively in s. 19 of the Industrial Relations and Disputes Investigation Act and art. 7.04 of the governing collective agreement. As a matter of history, collective agreements in Canada had no legal force in their own right until the advent of compulsory collective bargaining legislation. Our Courts refused to assume original jurisdiction for their enforcement and placed then outside of the legal framework within which contractual obligations of individuals were administered. The legislation, which is the context of encouragement to collective bargaining sought stability in employeremployee relations, envisaged arbitration through a mutually accepted tribunal as a built-in device for ensuring the resiliation of the rights and enforcement of the obligations which were the products of successful negotiation. Original jurisdiction without right of appeal was vested in boards of arbitration under legislative and consensual pres-

7 Voir en ce sens: Orphelinat de l'Immaculée-Conception, supra, note (1) à la p. 4 ; Syndicat des professeurs de l'État du Québec, supra, note (1), à la p. 242 ; affaire Rousseau, supra, note (1), à la p. 8; Compagnie Price Limitée, supra, note (2), à la page 572 .

8 Code civil, art. 1077 ; voir en particulier les deux premières décisions de la note (7).

9 Supra, note (3). 
criptions for finality and for binding determinations. In short, boards of arbitration were entrusted with a duty of effective adjudication differing in no way, save perhaps in the greater responsibility conferred upon them, from the adjudicative authority exercised by the ordinary Courts in civil cases of breach of contract. That the adjudication was intended to be remedial as well as declaratory could hardly be doubted. Expeditions settlement of grievances, without undue formality and without excessive cost, was no less a key to successful collective bargaining in day to day administration of collective agreements than the successful negotiation of the agreements in the first place. Favourable settlement where an employee was aggrieved meant not a formal abstract declaration of his rights and obligations of the collective agreement. In some jurisdiction, as for example, Ontario, this view was emphasized by the fact of statutory withdrawal of the application of Arbitration Acts from labour arbitrations, thus excluding the kind of curial review which was open to the parties to commercial arbitration. To have proposed to union negotiators that collective agreements, so long ignored in law and left to "lawless" enforcement by strikes and picketing, should continue to be merely empty vehicles for propounding declarations of right when the right to strike during their currency was taken away, would be to mock the policy of compulsory collective bargaining legislation which envisaged the collective agreement as the touchstone of the successful operation of that policy. ... The pivotal issue is simply whether the exercise of arbitral authority encompasses the effectuation of the right and the enforcement of the obligation which are submitted for both original and final adjudication. One would ordinarily think, especially if seised of any knowledge of the history of collective bargaining and its legislative implementation, that if there is any area of adjudication where abstract pronouncements, devoid of direction for redress of violations, would be unwelcome it would be in labour arbitration. Such alternation of arbitration authority must surely be found in explicit restrictions rather than in implicit limitation ".

Dans cette perspective, loin de rechercher dans la convention un fondement exprès, il faudrait plutôt que celle-ci ait exclu expressément ce pouvoir de l'arbitre, s'il ne devait pas pouvoir l'exercer, observe-t-on !

Combinant à la fois l'argument général tiré du droit civil des obligations à celui découlant du rôle de l'arbitre, le juge Lande, agissant comme arbitre concluait pour sa part ainsi récemment à des dommages-intérêts moratoires :

\& La Convention Collective est une convention comme toute autre convention et est assujettie aux mêmes règles. Dans une convention commerciale le débiteur qui enfreint son obligation est responsable de tout dommage qui en résulte, même si les sanctions n'y sont pas stipulées. Il n'est pas nécessaire que la convention collective stipule toutes les sanctions que l'arbitre peut appliquer en rendant ses sentences. Il est là pour redresser toute injustice et toute violation d'une obli- 
gation quelconque stipulée dans la convention. Or, la seule sanction qu'il lui reste en cas d'une violation d'une obligation est relativement aux dommages $\gg 10$.

De façon particulière, ajoutera-t-on, dans le cas de la justification de l'intérêt étant donné le retard à payer une somme due selon la convention, il ne s'agit que de l'accessoire du principal ${ }^{11}$.

Cette affirmation unanime, quoique diversement motivée, du caractère approprié des dommages-intérêts comme sanction de la convention, soulève le problème de la détermination de l'autorité compétente à les prononcer.

\section{QUI PEUT PRONONCER LES DOMMAGES-INTÉRÊTS ?}

Le tribunal de droit commun a, au Québec, revendiqué de façon exclusive de l'arbitre des griefs le pouvoir de sanctionner par des dommages-intérêts, une violation de la convention collective (A.) ce qui n'a cependant pas empêché des arbitres de les prononcer eux-mêmes, dans certains cas (B.).

\section{Le tribunal civil}

Dans l'arrêt Bédard-Girard ${ }^{12}$, la Cour d'appel a, en définitive, posé que le tribunal civil, avait, à l'exclusion de l'arbitre des griefs, la compétence d'accorder des dommages-intérêts, en réparation de la violation d'une clause de non-grève contenue dans la convention. Il est d'intérêt d'observer que l'arrêt ne fait qu'état de la violation de la convention pour établir la responsabilité du syndicat, sans faire allusion au fait que la grève en question était par ailleurs illégale en regard du Code du travail.

Le point de départ du raisonnement est évidemment la reconnaissance de la compétence juridictionnelle générale du tribunal de droit commun, la Cour supérieure, sous réserve seulement de la possibilité d'une attribution particulière de juridiction exclusive à un autre tribunal ${ }^{13}$. Mais, dira-t-on, le législateur n'a-t-il précisément pas confié les griefs à l'arbitre ? A cela, la Cour d'appel répond par une interprétation indûment restrictive, dirions-nous, de la définition de « grief » selon le Code du travail. Reprenant la conception du grief déjà énoncée dans les arrêts Maluorni ${ }^{14}$ et Association des policiers de Giffard ${ }^{15}$, elle réduit celui-ci aux seules mésententes relatives à l'interprétation de la convention collective ou à la détermination de son « aire d'applicabilité ». Partant, ne saurait, selon la Cour d'appel, être de la compétence de l'arbitre, une situation incontestée,

10 Affaire Rousseau, supra, note (1), à la p. 7 .

11 Syndicat des professeurs de l'État du Québec, supra, note (1), à la p. 242.

12 Supra, note (3).

13 Code de procédure civile, art. 31.

14 Maluorni v. Ville de Mont-Royal, (1969) B.R. 922 - commentaire P. Verge à (1969) 24 Relations industrielles 598.

15 Association des policiers de Giffard v. Cité de Giffard, (1968) B.R. 863, commentaire P. Verge à (1968) Relations industrielles 672. 
claire (du moins à ses yeux) de violation de la convention collective, comme dans l'espèce, c'est-à-dire qui ne soulève pas, notamment, une véritable difficulté d'interprétation de celle-ci. L'on entérine ici la constatation du jugement de première instance :

\begin{abstract}
"The Court", said he, "is quite unable to accept that the acts of the defendant, as alleged, constitute in any sense a disagreement respecting the interpretation or application of the collective agreement. What we have here is a violation of a clear provision relating to the execution of the agreement and not a disagreement as to its interpretation or application" 16 .
\end{abstract}

Ce dernier, par une exégèse de textes, qui semble abusive, avait, en effet, fait sienne une notion relativement restrictive du grief, selon le Code du travail, en excluant les situations de «violation » de la convention collective, à la différence notamment de la définition contenue dans la loi fédérale, qui était en cause dans l'arrêt rendu par la Cour suprême dans l'affaire Polymer.

Même si la définition contenue dans le Code du travail du Québec n'est pas absolument identique à celle que présente cette dernière loi, une 《 mésentente relative à l'application de la convention collective » vise précisément, à côté des cas d'interprétation de la convention, ceux de «violation » de celle-ci. Le législateur, dans l'un et l'autre cas, par des définitions en substances semblables, a confié exclusivement l'application (au sens ordinaire du terme, par opposition à la seule détermination de l'aire d'applicabilité de la convention) à l'arbitre des griefs. Partant, aurionsnous conclu, contrairement à l'arrêt Bédard-Girard, la sanction d'une violation de la convention par l'octroi de dommages-intérêts est du ressort, exceptionnellement, de l'arbitre des griefs, non du tribunal civil. Il est chargé expressément de se prononcer de façon finale sur les mésententes relatives à l'application de la convention.

Dans la même veine que l'arrêt Bédard-Girard, il faut également faire état d'une décision d'un tribunal d'arbitrage qui s'était refusé la compétence d'accorder des dommages-intérêts moratoires, toujours en considération de la place centrale, dans le système judiciaire québécois, du tribunal de droit commun ${ }^{17}$.

16 Jugement inédit, en date du 27 novembre 1968, C.S. (M.) no 756,045 (J. Mitchell), à la p. 7.

17 Décision Orphelinat de l'Immaculée-Conception de Chicoutimi, supra, note (1).

De même, dans une autre espèce, deux juges de la Cour d'Appel ont sans doute manifesté une semblable attitude, avant même que le tribunal d'arbitrage n'ait eu l'occasion d'ordonner la réparation: Association catholique des enseignants de l'Estrie v. Commission des écoles catholiques de Sherbrooke, (1970) C.A. 369, à la page 372 (res.) - commentaire F. Morin, (1969) 24 Relations industrielles 768. Fondamentalement, toutefois, l'on s'est alors prononcé sur l'étendue des pouvoirs du tribunal d'arbitrage en regard des pouvoirs de la direction. 
La divergence, on le voit, réside uniquement dans la portée qu'il y a lieu de reconnaître à l'attribution de compétence à l'arbitre en matière de griefs. Peut-être, également, bien qu'on ne l'ait pas exprimé ouvertement, l'affirmation de la compétence exclusive du tribunal civil tient-elle également au fait que, traditionnellement, celui-ci a toujours été chargé d'assurer le respect des obligations civiles, selon l'article 1065 du Code civil.

\section{L'arbitre des griefs}

L'arrêt Polymer ${ }^{18}$ de la Cour Suprême a affirmé, avons-nous vu, la compétence de l'arbitre de griefs, agissant selon la loi fédérale, de condamner à des dommages-intérêts un syndicat qui avait participé à un arrêt de travail contraire à la convention. C'est en faveur de l'arbitre que l'on énonça que le pouvoir décisionnel relatif à la convention se doit d'être efficace, l'arbitrage ayant été substitué de façon obligatoire par la loi au recours au lockout ou à la grève. C'est directement que l'on traita de la compétence de l'arbitre, et non indirectement, pour l'exclure, en posant celle du tribunal civil, comme dans l'arrêt Bédard-Girard.

Des décisions d'arbitres rendues selon le Code du travail, se sont autorisées de l'arrêt Polymer ${ }^{19}$, en dépit de ces divergences dans les définitions du « grief », qui ont retenu la Cour d'Appel dans Bédard-Girard.

Certes, il n'y a pas d'appel d'un arbitre octroyant des dommagesintérêts (sous réserve, cependant du contrôle par le tribunal de droit commun des pouvoirs de l'arbitre, ce qui est différent). Au contraire, un jugement du tribunal civil comporte généralement la possibilité d'un pourvoi en appel. Mais, il s'agit là d'une considération de politique législative, non d'une raison de nier la compétence de l'arbitre, si du moins, elle s'étend bien à la situation envisagée. De toute façon, le pouvoir de l'arbitre d'accorder des dommages-intérêts, dans les situations où cette conclusion est justifiée, découle de la loi, du caractère final et obligatoire de sa sentence en matière de grief (selon la définition qu'il est permis d'entretenir de ce dernier). Il ne saurait donc être question de vouloir subordonner son existence à une référence expresse à ce pouvoir dans la convention ${ }^{20}$.

18 Supra, note (3).

19 Affaire Rousseau, supra, note (1), à la p. 8; Syndicat des professeurs de l'État du Québec, ibidem, à la p. 242.

20 Voir notamment les décisions mentionnées dans la note précédente. Contra, décision Orphelinat de l'Immaculée-Conception de Chicoutimi, supra, note (1).

Observons en passant que la décision Syndicat des professeurs de l'État du Québec est celle d'un arbitre qui accorde des dommages-intérêts moratoires à la suite d'une première décision d'un autre arbitre, lequel s'était, semble-t-il, limité à conclure relativement à l'exigibilité de la somme principale. Le grief ne se trouvait-il pas à avoir été «vidé » - quoique incomplètement -, par le premier arbitre, devenu lui-même functus officio ? N'était-on pas déjà parvenu au stade de l'exécution par le tribunal civil de la sentence, selon l'art. 89 du Code du travail, dès cette décision initiale? 